\title{
MONOCLONAL ANTIBODY AGAINST CADHERIN-17 AS A POTENTIAL TREATMENT FOR LIVER CANCER
}

\author{
N.P. Lee ${ }^{1}$, Y. Wang ${ }^{2}$, J.M. Luk ${ }^{3}$ \\ ${ }^{1}$ Surgery, The University of Hong Kong, Hong Kong, Hong Kong S.A.R.; ${ }^{2}$ Oncology, Shanghai \\ Jiao Tong University, Shanghai, China; ${ }^{3}$ Pharmacology and Surgery, National University Health \\ System, Singapore
}

BACKGROUND AND AIMS: Hepatocellular carcinoma (HCC) is a major type of liver cancer associated with high mortality. Prognosis is poor in HCC patients largely because of late diagnosis and limitations in treatment options. Therefore, this study aims to identify alternative target for HCC in hope to offer new treatments to patients. Cadherin-17 (CDH17) has been identified as an oncofetal molecule of HCC and that a suppression of its expression by RNA interference (RNAi) leads to anti-tumorigenesis. To supplement the drawbacks associated with the use of RNAi approach in biotherapy, we developed specific antibody against CDH17 for achieving similar purpose. METHODS: Hybridoma cell clones capable of secreting antibodies against ectodomains of human CDH17 were injected subcutaneously into nude mice to stimulate ascites production, after which monoclonal antibody against CDH17 (anti-CDH17) was tested on purity, specificity and immunogenicity. In vitro functional assays were conducted to assess the effect of anti-CDH17 treatment in altering the tumor phenotypes of HCC cells. Besides, subcutaneous tumor xenograft model was developed for examining the tumor-suppressing capacities of anti-CDH17 treatment alone or in combined with chemotherapeutic drug cisplatin. For tumor xenografts from anti-CDH17 treatment group, biochemical analyses examining cell proliferation, extent of apoptosis and effects on CDH17-b-catenin axis were analyzed. RESULTS: Anti-CDH17 derived from mouse ascites associated with high purity, specificity and immunogenicity in detecting CDH17 and demonstrated a profound effect in suppressing CDH17. Treatment of anti-CDH17 suppressed proliferation, migration, invasion and colony formation of cultured CDH17-expressing HCC cells (MHCC97L/97L and MHCC97H/97H), but not CDH17deficient cells. When mice bearing 97L-derived subcutaneous tumors were treated with anti$\mathrm{CDH} 17$, regression in tumor sizes accompanying with less chance of developing lung metastasis were observed and more prominent effects were resulted in combined with cisplatin. This antitumor effect of anti-CDH17 treatment was linked to an inactivation of the CDH17-b-catenin axis frequently found in CDH17-expressing HCC. Tumor xenografts subjected to anti-CDH17 treatment associated with a reduced tumor cell proliferation and an increased level of apoptosis. CONCLUSIONS: Anti-CDH17 treatment inactivates CDH17-b-catenin axis and leads to antitumorigenic effects in $\mathrm{CDH} 17$-expressing HCC cells and xenografts, suggesting its future development as alternative medicine for HCC. 